Pathologe 2018 - 39 (Suppl 2):S352 https://doi.org/10.1007/s00292-018-0556-1 Online publiziert: 7. Dezember 2018 (c) Springer Medizin Verlag $\mathrm{GmbH}$, ein Teil von Springer Nature 2018

CrossMark

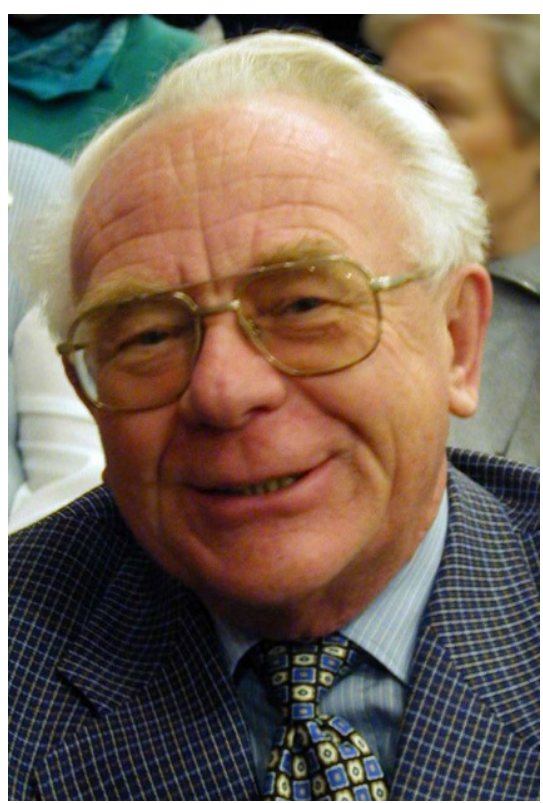

Gerhard Korb

Am 24. Dezember 2017 verstarb in seinem Alterssitz in München Professor Dr. Gerhard Korb nach langem, wissenschaftlich und persönlich erfolgreichem Leben im 88. Lebensjahr.

Gerhard Korb wurde am 9. Februar 1929 in Iglau/Mähren geboren und studierte Medizin an der Humboldt-Universität in Berlin. Nach Abschluss seines Studiums wandte sich Gerhard Korb dem Fach Pathologie zu. Er wirkte an der Charité in Berlin sowie als Professor in Marburg und Regensburg. Schließlich gründete er 1967 am Klinikum Weiden ein Institut für Pathologie, welches sich innerhalb kurzer Zeit einen hervorragenden Ruf als klinisch orientierte Anstalt mit hoher fachlicher Kompetenz erwarb. Sein weiteres besonderes Augenmerk widmete er dem Aufbau und der Führung der Weidener Krankenpflegeschule.

\title{
H. Denk
}

Institut für Pathologie, Medizinische Universität Graz, Graz, Österreich

\section{Gerhard Korb}

\subsubsection{9-24.12.2017}

Im wissenschaftlichen Bereich galt anfangs sein Interesse der Kardiologie, nach einem Studienaufenthalt in New York, im damaligen „Mekka“ der Hepatopathologie, faszinierte ihn die Leber mit allen ihren klinischen und pathologisch-morphologischen Aspekten. Auf diesem Gebiet hat er sich hohes internationales Ansehen erworben und unser Wissen sehr bereichert. Die für die klinische Betreuung der Patienten relevante Aussagekraft morphologischer Befunde war ihm ein besonderes Anliegen. Die internationale Anerkennung und Wertschätzung des Hepatopathologen Korb zeigte sich nicht nur in den zahlreichen an ihn ergangenen Vortragseinladungen aus aller Welt, sondern auch in der Tatsache, dass er 1967 als Gründungsmitglied einer internationalen Gruppe spezialisierter Leberpathologen fungierte, die unter dem Namen „Gnomes“ bis heute Standards in der Leberbiopsiediagnostik vorgibt. Drei Arbeitstreffen mit den Schwerpunkten "Hepatitis“ (1973), „Gallengangsläsionen“ (1980) und „systemische Lebererkrankungen/ Lebergranulome“ (1990) wurden von ihm organisiert und geleitet.

Wir verdanken Gerhard Korb über 120 Publikationen, wobei besonders seine Arbeiten zur Hepatitisthematik große internationale Beachtung gefunden haben.

Wir werden Gerhard Korb als geschätzten, im persönlichen Leben und in seiner Wissenschaft erfolgreichen, vorbildlichen und tatkräftigen Kollegen in steter Erinnerung behalten.

\section{Helmut Denk}

Graz

\section{Korrespondenzadresse}

Prof. Dr. H. Denk

Institut für Pathologie, Medizinische Universität Graz

Neue Stiftingtalstraße 6, 8010 Graz, Österreich helmut.denk@medunigraz.at 